\title{
Network Pharmacology Analysis Uncovers the Potential Anti- Hypertensive Mechanisms of Xia Sang Ju Granule
}

\author{
Minhua Peng ${ }^{1,2^{*}}$ \\ ${ }^{1}$ Shenzhen International Institute for Biomedical Research, 518116 Shenzhen, Guangdong, China; \\ ${ }^{2}$ Shenzhen Institutes of Advanced Technology, Chinese Academy of Sciences, 518055 Shenzhen, Guangdong, China
}

\begin{abstract}
Background and objectives: Xia Sang Ju (XSJ) granule, a Chinese drug and herbal tea made up of Prunellae spica (Xia Ku Cao), Mori folium (Sang Ye), and Flos Chrysanthemi Indici (Ye Ju Hua), is commonly used for fever, headache, and sore throat. The underlying pharmacological mechanism of XSJ on hypertension treatment is described here, based on network pharmacology.

Methods: The compounds in XSJ were searched using the Traditional Chinese Medicine Systems Pharmacology Database and Analysis Platform (commonly known as TCMSP), and the active components, according to oral bioavailability and drug likeness, were screened. Compounds targets were predicted by the SwissTargetPrediction web server, while hypertension targets were collected from the Online Mendelian Inheritance in Man (commonly known as OMIM) and GeneCards databases. The interaction of targets was analyzed by STRING. The compoundcompound target network was constructed by Cytoscape. Gene Ontology enrichment and Kyoto Encyclopedia of Genes and Genomes (commonly known as KEGG) pathways were analyzed by the Database for Annotation, Visualization and Integrated Discovery (commonly known as DAVID).
\end{abstract}

Results: Forty-five active compounds were obtained from 359 ingredients present in the XSJ decoction, corresponding to 237 targets. In addition, 189 genes were found to be related to hypertension, of which 11 overlapped with XSJ targeted by 28 compounds and were thus considered therapeutically-relevant. ESR 2 was the most frequent gene targeted by the compounds, while NR3C1 showed the most interaction with other genes. These results revealed that the anti-hypertensive activity of XSJ may directly relate to the regulation of several hypertension-associated biological processes and pathways, such as cellular nitrogen compound biosynthetic process, positive regulation of the nitrogen compound metabolic process, steroid hormone biosynthesis, and aldosteroneregulated sodium reabsorption.

Conclusions: These findings provide a reference for further interpretation of the potential mode of action of XSJ against hypertension and serve as an example for elucidation of the Traditional Chinese Medicine concept of "multiple compounds-multiple targets-multiple effects".

Keywords: Xia Sang Ju granule; Hypertension; Network pharmacology; Multiple compounds-multiple targets-multiple effects.

Abbreviations: DAVID, Database for Annotation, Visualization and Integrated Discovery; KEGG, Kyoto Encyclopedia of Genes and Genomes; OB, oral bioavailability; DL, drug likeness; OMIM, Online Mendelian Inheritance in Man; SMILES, simplified molecular-input line-entry system; TCMSP, Traditional Chinese Medicine Systems Pharmacology Database and Analysis Platform; TCM, traditional Chinese medicine; XSJ, Xia Sang Ju.

Received: April 22, 2020; Revised: May 16, 2020; Accepted: May 23, 2020

*Correspondence to: Minhua Peng, Shenzhen International Institute for Biomedical Research, 518116 Shenzhen; Shenzhen Institutes of Advanced Technology, Chinese Academy of Sciences, 518055 Shenzhen, Guangdong, China. Tel: +86-13527635482, Fax: +86-0755-28291883, E-mail: pengminhua0921@foxmail.com

How to cite this article: Peng M. Network Pharmacology Analysis Uncovers the Potential Anti-Hypertensive Mechanisms of Xia Sang Ju Granule. J Explor Res Pharmacol 2020;5(4):51-60. doi: 10.14218/JERP.2020.00008.
Introduction

Xia Sang Ju (XSJ) granule is a traditional Chinese drug as well as a kind of Chinese herbal tea which is made up of Prunellae spica (Xia Ku Cao), Mori folium (Sang Ye), and Flos Chrysanthemi Indici (Ye Ju Hua). It originates from the classic prescription called Sang Ju Yin that was recorded in the Treatise on Differentiation and Treatment of Epidemic Febrile Diseases (Wen Bing Tiao Bian) by Wu Jutong in the Qing Dynasty. Though XSJ is well-known for treating fever, headache, and sore throat, hypertension is also one of the main functions of XSJ. ${ }^{1}$ However, how XSJ plays a part in anti-hypertensive activity remains 
unclear, due to the complexity of Traditional Chinese medicine (TCM).

Hypertension is characterized by elevated blood pressure in arteries, and is the most common of the chronic diseases and one of the most important risk factors for cerebrovascular diseases; causing an estimated 7.5 million deaths, it accounts for $12.8 \%$ of the total deaths. ${ }^{2}$ So far, commonly-used anti-hypertensive drugs include diuretics, beta-blockers, angiotensin converting enzyme inhibitors, calcium channel blockers, angiotensin receptor blockers, etc. ${ }^{3}$ Unfortunately, no specific medicine can yet cure high blood pressure.

TCMs are extensively used in eastern countries, as treatments for such chronic diseases as hypertension, diabetes and stroke, and their advantages have been gradually recognized through the increasing number of people who seek natural herbal remedies in western countries. ${ }^{4}$ Most existing research is limited to a certain gene target while interpreting the mechanism of a drug, an approach which may ignore the multi-component, multi-target, multi-pathway characteristics of Chinese herbal formulae. ${ }^{4}$ Network pharmacology, based on an integrated multidisciplinary concept, is a powerful tool that analyzes the multi-level network of molecular-target-pathway-disease through the interaction between TCM and disease from a holistic perspective. ${ }^{5-9}$

In this study, firstly the active compounds of XSJ were screened computationally, according to oral bioavailability (OB) and drug likeness (DL) ${ }^{10}$ and then the potential compound targets and hypertension-related targets were predicted. Finally the XSJ-compound-hypertension networks were constructed, so as to deeply understand the potential underlying mechanism of the anti-hypertensive effect of XSJ.

\section{Materials and methods}

\section{Compounds of XSJ}

The Traditional Chinese Medicine Systems Pharmacology Database and Analysis Platform (commonly known as TCMSP; http:// www.tcmspw.com/tcmsp.php, version 2.3 $)^{11}$ was used to collect the compound information of XSJ. A total of 60 compounds in Prunellae spica, 269 compounds in Mori folium, and $30 \mathrm{com}-$ pounds in Flos Chrysanthemi Indici were found. To select the potential active compounds, $\mathrm{OB}$ and $\mathrm{DL},{ }^{10}$ the most important criteria for drug screening, was set to be $\geq 30 \%$ and $\geq 0.18$, respectively. ${ }^{12}$

\section{Compound targets}

To predict the most relevant targets of compounds, the simplified molecular-input line-entry system (referred to as SMILES) format of each compound was input into the SwissTargetPrediction website (http://www.swisstargetprediction.ch/) with the organism limited to Home sapiens. ${ }^{13,14}$

\section{Hypertension targets}

Genes associated with hypertension were searched from the Online Mendelian Inheritance in Man (commonly known as OMIM) database (http://www.omim.org/) (15,16 $^{16}$ and GeneCards database (https://www.genecards.org/) ${ }^{17}$ using the keywords "hypertension" or "high blood pressure".

\section{Protein-protein interaction}

The STRING database (https://string-db.org/, version 10.5) was used to analysis the protein-protein interaction. ${ }^{18}$ Protein names were input and organism was limited to Homo sapiens. Data of protein-protein interactions were obtained and saved as TSV files.

\section{GeneMANIA analysis}

A weighted composite functional interaction network for hypertension-related genes were constructed by GeneMANIA (https:// genemania.org/). ${ }^{19}$ Genes of interest were input and organism was limited to Homo sapiens.

\section{Network construction}

All the networks were constructed by Cytoscape software (https:// cytoscape.org/, version 3.6.1). ${ }^{20}$

\section{Gene ontology enrichment analysis}

Gene ontology enrichment analysis for biological processes and Kyoto Encyclopedia of Genes and Genomes (commonly known as KEGG) pathways were performed by Database for Annotation, Visualization and Integrated Discovery, commonly known as DAVID, 6.8 server (https://david.ncifcrf.gov/). ${ }^{21,22}$

Results

\section{Screen of active compounds}

In total, 359 compounds in XSJ were obtained from the TCMSP database. After filtering by $\mathrm{OB}$ and drug likeness parameters, 11 compounds from Prunellae spica, 29 compounds from Mori folium, and 12 compounds from Flos Chrysanthemi Indici with favorable pharmacokinetic profiles were included for further investigation (Table 1). Specifically, beta-sitosterol and quercetin were found in all three of the herbs, and kaempferol as well as stigmasterol were originated from both Prunellae spica and Mori folium, while luteolin was found in Prunellae spica and Flos Chrysanthemi Indici.

\section{Hypertension network analysis}

In total, 189 genes associated with hypertension were obtained from the OMIM and GeneCards databases after elimination of false positives and repetitive genes (Table s1). The interaction of hypertension target genes was analyzed by GeneMANIA (Fig. 1, Table s2) and a network containing 274 nodes and 10,742 edges was constructed. This result showed that $55.08 \%$ of genes were co-expressed, and $20.87 \%$ were expressed in the same tissue or their products in the same cellular location. Among the genes, $11.02 \%$ were found to be involved in physical interaction, while $4.86 \%$ were engaged in predicted functional relationships. Up to $3.61 \%$ were identified as possibly participating in the same pathway, $3.01 \%$ had shared protein domains, and $1.55 \%$ had genetic interactions that were functionally associated. 
Table 1. Active compounds in the herbs and their properties

\begin{tabular}{|c|c|c|c|c|}
\hline Mol ID & Compound & OB, \% & DL & Herbs \\
\hline MOL000358 & Beta-sitosterol & 36.91 & 0.75 & $\begin{array}{l}\text { Prunellae spica, Mori folium, } \\
\text { Flos Chrysanthemi Indici }\end{array}$ \\
\hline MOL000422 & Kaempferol & 41.88 & 0.24 & Prunellae spica, Mori folium \\
\hline MOL004355 & Spinasterol & 42.98 & 0.76 & Prunellae spica \\
\hline MOL000449 & Stigmasterol & 43.83 & 0.76 & Prunellae spica, Mori folium \\
\hline MOL004798 & Delphinidin & 40.63 & 0.28 & Prunellae spica \\
\hline MOL000006 & Luteolin & 36.16 & 0.25 & Prunellae spica, Flos Chrysanthemi Indici \\
\hline MOL006767 & Vulgaxanthin-I & 56.14 & 0.26 & Prunellae spica \\
\hline MOL006772 & Poriferasterol monoglucoside_qt & 43.83 & 0.76 & Prunellae spica \\
\hline MOL006774 & Stigmast-7-enol & 37.42 & 0.75 & Prunellae spica \\
\hline MOL000737 & Morin & 46.23 & 0.27 & Prunellae spica \\
\hline MOL000098 & Quercetin & 46.43 & 0.28 & $\begin{array}{l}\text { Prunellae spica, Mori folium, } \\
\text { Flos Chrysanthemi Indici }\end{array}$ \\
\hline MOL001771 & Poriferast-5-en-3beta-ol & 36.91 & 0.75 & Morifolium \\
\hline MOL002218 & Scopolin & 56.45 & 0.39 & Mori folium \\
\hline MOL002773 & Beta-carotene & 37.18 & 0.58 & Mori folium \\
\hline MOL003842 & Albanol & 83.16 & 0.24 & Mori folium \\
\hline MOL003847 & Inophyllum E & 38.81 & 0.85 & Mori folium \\
\hline MOL003850 & 26-Hydroxy-dammara-20,24-dien-3-one & 44.41 & 0.79 & Mori folium \\
\hline MOL003851 & Isoramanone & 39.97 & 0.51 & Mori folium \\
\hline MOL003856 & Moracin B & 55.85 & 0.23 & Mori folium \\
\hline MOL003857 & Moracin C & 82.13 & 0.29 & Mori folium \\
\hline MOL003858 & Moracin D & 60.93 & 0.38 & Morifolium \\
\hline MOL003859 & Moracin E & 56.08 & 0.38 & Morifolium \\
\hline MOL003860 & Moracin F & 53.81 & 0.23 & Mori folium \\
\hline MOL003861 & Moracin G & 75.78 & 0.42 & Mori folium \\
\hline MOL003862 & Moracin $\mathrm{H}$ & 74.35 & 0.51 & Mori folium \\
\hline MOL003879 & 4-Prenylresveratrol & 40.54 & 0.21 & Morifolium \\
\hline MOL000433 & & 68.96 & 0.71 & Mori folium \\
\hline MOL000729 & Oxysanguinarine & 46.97 & 0.87 & Morifolium \\
\hline MOL001439 & Arachidonic acid & 45.57 & 0.2 & Mori folium \\
\hline MOL001506 & Supraene & 33.55 & 0.42 & Mori folium \\
\hline MOL003759 & Iristectorigenin A & 63.36 & 0.34 & Mori folium \\
\hline MOL003975 & Icosa-11,14,17-trienoic acid methyl ester & 44.81 & 0.23 & Mori folium \\
\hline MOL006630 & Norartocarpetin & 54.93 & 0.24 & Mori folium \\
\hline MOL007179 & Linolenic acid ethyl ester & 46.1 & 0.2 & Mori folium \\
\hline MOL007879 & Tetramethoxyluteolin & 43.68 & 0.37 & Mori folium \\
\hline MOL013083 & Skimmin $(8 \mathrm{Cl})$ & 38.35 & 0.32 & Mori folium \\
\hline MOL001689 & Acacetin & 34.97 & 0.24 & Flos Chrysanthemi Indici \\
\hline MOL001790 & Linarin & 39.84 & 0.71 & Flos Chrysanthemi Indici \\
\hline MOL000359 & Sitosterol & 36.91 & 0.75 & Flos Chrysanthemi Indici \\
\hline MOL008173 & Daucosterol_qt & 36.91 & 0.75 & Flos Chrysanthemi Indici \\
\hline
\end{tabular}


Table 1. Active compounds in the herbs and their properties - (continued)

\begin{tabular}{|c|c|c|c|c|}
\hline Mol ID & Compound & OB, \% & DL & Herbs \\
\hline MOL008915 & Acacetin-7-O- $\beta$-D-galactopyranoside & 50.19 & 0.77 & Flos Chrysanthemi Indici \\
\hline MOL008918 & Arteglasin A & 52.45 & 0.33 & Flos Chrysanthemi Indici \\
\hline MOL008919 & $\begin{array}{l}\text { (2S,6S,7aR)-2-[(1E,3E,5E,7E,9E,11E,13E,15E)- } \\
\text { 16-[(4S)-4-Hydroxy-2,6,6-trimethyl-1- } \\
\text { cyclohexenyl]-1,5,10,14-tetramethylhexadeca- } \\
\text { 1,3,5,7,9,11,13,15-octaenyl]-4,4,7a-trimethyl- } \\
\text { 2,5,6,7-tetrahydrobenzofuran-6-ol }\end{array}$ & 59.52 & 0.55 & Flos Chrysanthemi Indici \\
\hline MOL008924 & $\begin{array}{l}\text { Azuleno(4,5-b)furan-2(3H)-one, 4-(acetyloxy)- } \\
\text { 3a,4,5,6,6a,7,9a,9b-octahydro-6-hydroxy- } \\
\text { 6,9-dimethyl-3-methylene-, (3aR-(3aalpha, } \\
\text { 4alpha,6alpha,6aalpha,9aalpha,9bbeta))- }\end{array}$ & 68.44 & 0.27 & Flos Chrysanthemi Indici \\
\hline MOL008925 & $\begin{array}{l}\text { (3aR,4S,6R,6aR,9aR,9bR)-4,6-Dihydroxy- } \\
\text { 6,9-dimethyl-3-methylene-4,5,6a,7,9a,9b- } \\
\text { hexahydro-3aH-azuleno[5,4-d]furan-2-one }\end{array}$ & 40.08 & 0.19 & Flos Chrysanthemi Indici \\
\hline
\end{tabular}

DL, drug likeness.

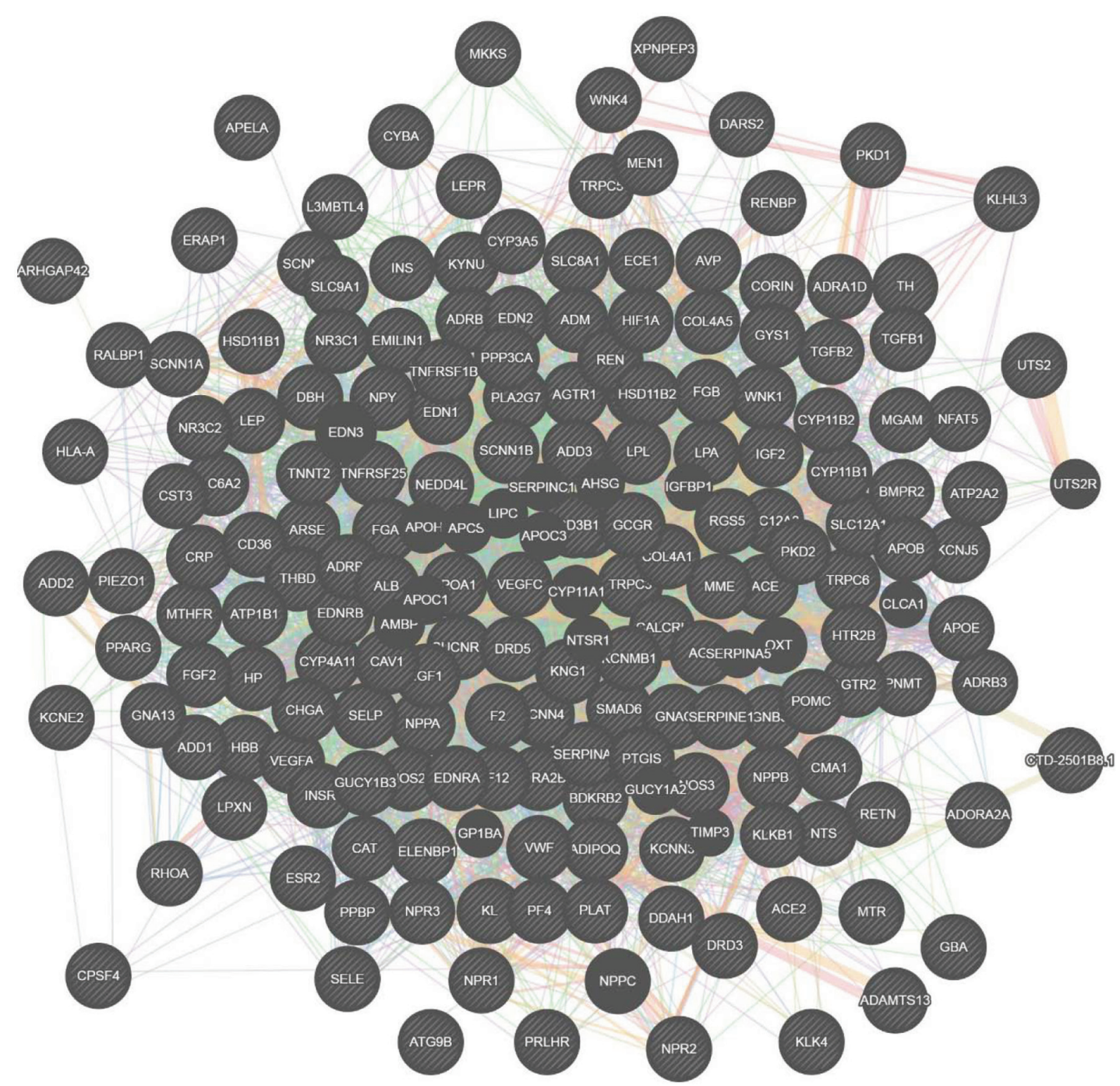

Fig. 1. Protein-protein interaction network of hypertension targets. 


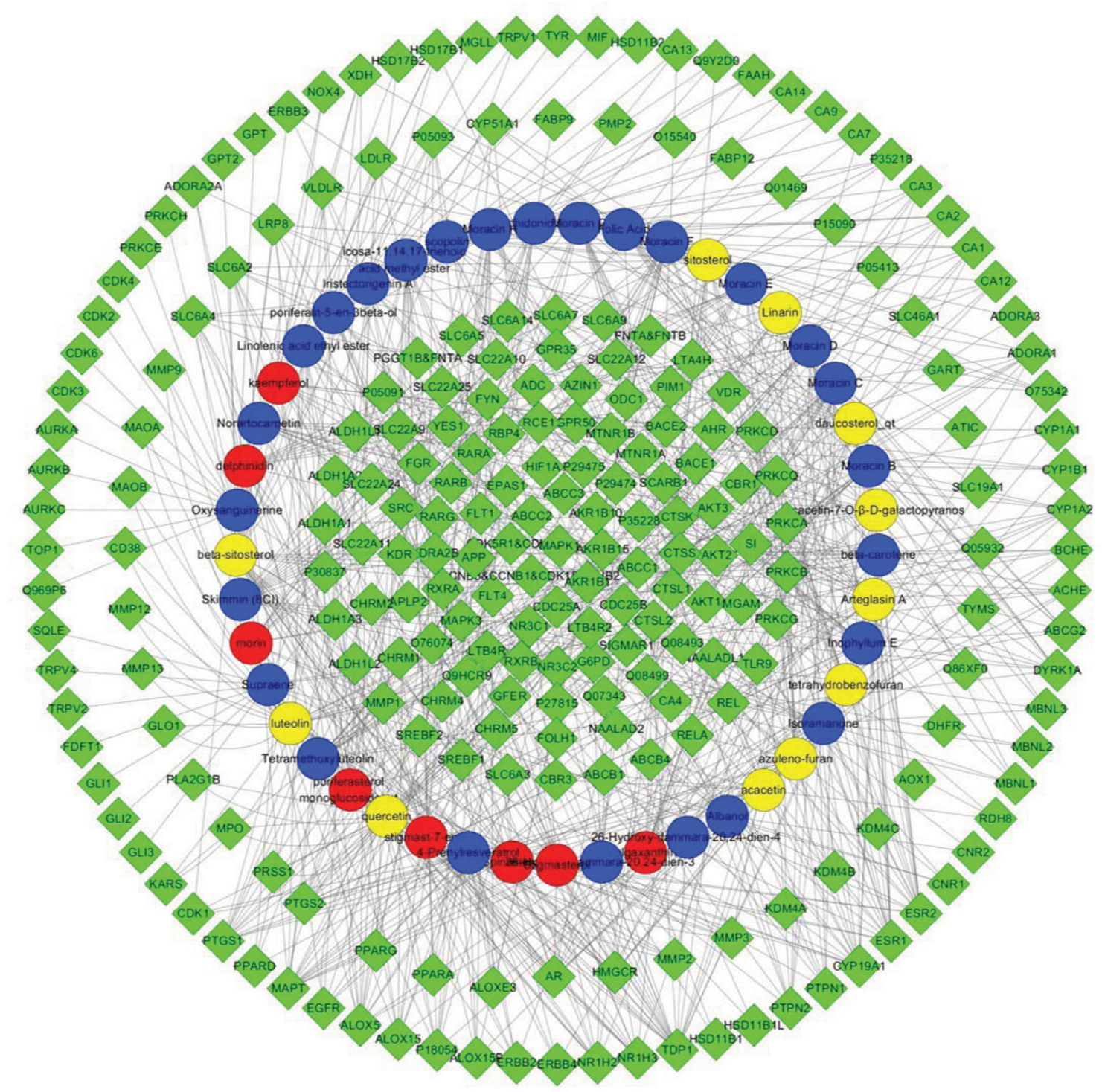

Fig. 2. Compound-compound target network of Xia Sang Ju (XSJ). Compound targets are denoted by green hexagons; Prunellae spica by blue circles; Mori folium by red circles; Flos Chrysanthemi Indici by yellow circles. "Azuleno(4,5-b)furan-2(3H)-one, 4-(acetyloxy)-3a,4,5,6,6a,7,9a,9b-octahydro-6-hydroxy-6,9-dimethyl-3-methylene-, (3aR-(3aalpha,4alpha,6alpha,6aalpha,9aalpha,9bbeta))" is abbreviated to "Azuleno-furan"; "(2S,6S,7aR)-2-[(1E,3E,5E,7E,9E,11E,13E,15E)-16-[(4S)-4-hydroxy-2,6,6-trimethyl-1-cyclohexenyl]-1,5,10,14-tetramethylhexadeca-1,3,5,7,9,11,13,15-octaenyl]-4,4,7a-trimethyl-2,5,6,7-tetrahydrobenzofuran-6-ol)" is abbreviated to "tetrahydrobenzofuran"; "(3aR,4S,6R,6aR,9aR,9bR)-4,6-dihydroxy-6,9-dimethyl-3-methylene-4,5,6a,7,9a,9b-hexahydro-3aH-azuleno[5,4-d]furan-2-one" is abbreviated to "azuleno-furan".)

\section{Analysis of compound-compound target network}

The SMILES format of each compound was input into SwissTargetPrediction, and predicted compound targets were obtained (Table s3). A compound-compound target network was constructed, consisting of 282 nodes and 703 edges (Fig. 2). These results showed that some target genes may be modulated by many compounds, such as the ESR1, AR, MAPT, CYP19A1, and HMGCR genes. While the $A O X 1, C T S K, O C D 1, S R C, R A R A, N O X 4$, and CDC25B genes are hit by only one compound. Interestingly, both SLC6A4 and P05093 can be regulated by poriferast-5-en-3beta-ol, beta-sitosterol, poriferasterol mo noglucoside qt, stigmast-7-enol, spinasterol, daucosterol qt, and sitosterol. Both the NR1H2 and NR1H3 genes can be modulated by 26-hydroxy-dammara-20,24-dien-3, poriferast-5-en3 beta-ol, beta-sitosterol, poriferasterol monoglucoside qt, stigmast7-enol, spinasterol, stigmasterol, daucosterol qt, and sitosterol. This predicted compound-compound target network strengthens the concepts of multi-compound-multi-target of TCM, in which different active components in XSJ may regulate the same targets and one active ingredient may also modulate various targets.

\section{Hypertension-related compound target network analysis}

Eleven genes with commonalities between hypertension genes and compound targets were found and a hypertension-related compound 


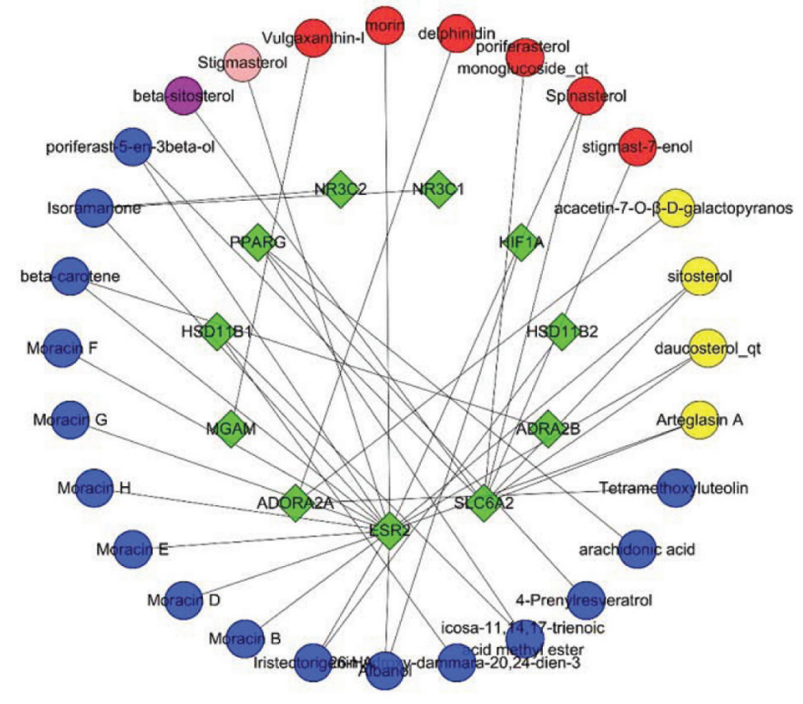

Fig. 3. XSJ-hypertension network. Compound targets and hypertension targets are denoted by green hexagons; Prunellae spica by blue circles; Mori folium by red circles; Flos Chrysanthemi Indici by yellow circles; Prunellae spica and Mori folium by pink circle; Prunellae spica and Mori folium and Flos Chrysanthemi Indici by purple circle.

target network was constructed (Fig. 3, Table 2), which contained 39 nodes and 39 edges. Among the 28 compounds directly interacting with these genes, 8 of them came from Prunellae spica, 18 were from Mori folium, and 5 were from Flos Chrysanthemi Indici. The protein classes for the 11 common genes were obtained from the DisGeNET database. The XSJ and hypertension-related targets' protein-protein interaction network is shown in Figure 4. ESR2 and $S L C 6 A 2$, both of which play a role in nucleic acid binding, as receptor and transcription factor, or transporter, were the most frequent genes targeted by the compounds. ESR2 and SLC6A2 are known to be important to cardiovascular physiology and blood pressure regulation. ${ }^{23-27}$ These results suggested that the anti-hypertension effect of XSJ may be regulated mainly by ESR2 and SLC6A2 (Table 3).

\section{Biological functional analysis}

Biological functions of the hypertension-related compound targets were annotated to explain the possible mode of action of XSJ in hypertension. Gene ontology enrichment analysis was performed on the 11 targets by DAVID. The top five biological processes were cellular nitrogen compound biosynthetic process, organic cyclic compound biosynthetic process, aromatic compound biosynthetic process, heterocycle biosynthetic process, and nucleobase-containing compound biosynthetic process (Fig. 5a). The significant KEGG pathways included neuroactive ligand-receptor interaction, steroid hormone biosynthesis, aldosterone-regulated sodium reabsorption, PPAR signaling pathway, and thyroid cancer (Fig. 5b). These results elucidated that XSJ may exert anti-hypertension activity through multi-biological processes as well as multi-pathways.

\section{Discussion}

The escalation of hypertension cases global effects. Coupled with lack of any promising hypotensor, this then requires multiple ap- proaches for treatment, including lifestyle modifications and new drugs. Though XSJ is generally used for treatment of fever, headache, sore throat, and as a beverage for clearing heat, hypertension is also one of the major functions. ${ }^{1}$ Nevertheless, the mechanism of action for XSJ working on hypertension remains to be fully understood.

During the development of hypertension, endothelin, nitric oxide, and angiotensin II are key factors. Vascular endothelial cells can produce both systolic and vasoactive substances for maintaining vasomotor balance and normal tension. Endothelin is the strongest vasoconstrictor and promotes smooth muscle proliferation, ${ }^{28}$ while nitric oxide is the main vasodilator substance released by vascular endothelial cells. Endothelin harbors angiotensin-converting enzyme activity that catalyzes the synthesis of angiotensin II; however, angiotensin II can induce expression of the endothelin gene in endothelial cells. Nitric oxide inhibits the production and release of endothelin, and also inhibits the release of renin, which in turn inhibits the production of angiotensin II. ${ }^{29-31}$

Despite few publications in the publicly available literature describing the anti-hypertension activity of XSJ so far, recent studies have proven that the extracts and some compounds of all three herbs in XSJ have direct or indirect anti-hypertensive effect, consistent with some of the biological processes found in our study. Ethanol extract of Prunella vulgaris $L$ has been shown to increase the content of nitric oxide, to decrease the content of endothelin and angiotensin II, and finally to reduce blood pressure significantly in a spontaneously hypertensive rat model (e.g., positive regulation of the nitric oxide biosynthetic process, regulation of the systemic arterial blood pressure by endothelin). ${ }^{32}$ Flavonoid compounds in Mori folium have also been found to expand the coronary vessels, improve myocardial circulation, and reduce blood pressure (e.g., regulation of blood pressure).$^{33}$ Ethanol extract of Flos Chrysanthemi Indici has shown hypotensive effect in clinical studies. ${ }^{34}$ Intriguingly, luteolin from Prunella vulgaris $L$ and Flos Chrysanthemi Indici might inhibit vascular smooth muscle cell proliferation and migration, which is pivotal in the development of arterial remodeling during hypertension (e.g., blood vessel remodeling), by suppressing transforming growth factor- $\beta$ receptor 1 signaling. ${ }^{35}$

Luteolin can ameliorate hypertensive vascular remodeling through inhibition of proliferation and migration of angiotensin IIinduced vascular smooth muscle cells, a process that is mediated by the regulation of MAPK signaling pathway and the production of reactive oxygen species (e.g., blood vessel remodeling, positive regulation of the reactive oxygen species metabolic process) ${ }^{36}$ Quercetin from Prunella vulgaris L and Flos Chrysanthemi Indici can attenuate hypertension via reduction in oxidative stress and improving endothelial function, as shown in an acute fluorideinduced hypertension and cardiovascular complications model. ${ }^{37}$ Furthermore, quercetin was shown to reduce hypertension-induced vascular remodeling, oxidative stress and MMP-2 activity in aortas in the two-kidney one-clip hypertensive Wistar rat model (e.g., blood vessel remodeling). ${ }^{38}$ Quercetin can also attenuate vascular contraction through the LKB1-AMPK signaling pathway (e.g. regulation of vasoconstriction). ${ }^{39}$ Delphinidin and quercetin were shown to block the renin-angiotensin system signaling pathway through inhibition of angiotensin-converting enzyme activity and decreasing the production of its mRNA. ${ }^{40}$ Finally, linarin from Flos Chrysanthemi Indici was shown to directly or indirectly activate macrophages and affect the inhibition of nitric oxide that is responsible for vasodilation and hypotension (e.g., vasodilation) ${ }^{41}$

The current study provided a prediction of the potential mechanism of XSJ as treatment of hypertension, based on a computational approach. There are some limitations of this work. First, the 
Table 2. Candidate compounds from Xia Sang Ju and their potential targets associated with hypertension

\begin{tabular}{|c|c|c|c|}
\hline No. & Compound & Target gene code & Herbs \\
\hline 1 & Stigmast-7-enol & SLC6A2 & Prunellae spica \\
\hline 2 & Spinasterol & SLC6A2, ESR2 & Prunellae spica \\
\hline 3 & Poriferasterol monoglucoside_qt & SLC6A2 & Prunellae spica \\
\hline 4 & Delphinidin & ADORA2A & Prunellae spica \\
\hline 5 & Morin & ESR2 & Prunellae spica \\
\hline 6 & Stigmasterol & ESR2 & Prunellae spica, Mori folium \\
\hline 7 & Vulgaxanthin-I & MGAM & Prunellae spica \\
\hline 8 & Beta-sitosterol & SLC6A2 & Prunellae spica, Mori folium, Flos Chrysanthemi Indici \\
\hline 9 & Poriferast-5-en-3beta-ol & SLC6A2, ESR2 & Mori folium \\
\hline 10 & Isoramanone & $E S R 2, N R 3 C 2, N R 3 C 1$ & Mori folium \\
\hline 11 & Beta-carotene & $A D R A 2 B, E S R 2$ & Mori folium \\
\hline 12 & Moracin F & ESR2 & Mori folium \\
\hline 13 & Moracin G & ESR2 & Mori folium \\
\hline 14 & Moracin $\mathrm{H}$ & ESR2 & Mori folium \\
\hline 15 & Moracin E & ESR2 & Mori folium \\
\hline 16 & Moracin D & ESR2 & Mori folium \\
\hline 17 & Moracin B & ESR2 & Mori folium \\
\hline 18 & Iristectorigenin A & ESR2, HSD11B2 & Mori folium \\
\hline 19 & Albanol & HIF1A, ESR2 & Mori folium \\
\hline 20 & 26-Hydroxy-dammara-20,24-dien-3 & HSD11B1 & Mori folium \\
\hline 21 & $\begin{array}{l}\text { Icosa-11,14,17-trienoic } \\
\text { acid methyl ester }\end{array}$ & HSD11B1, PPARG & Mori folium \\
\hline 22 & 4-Prenylresveratrol & PPARG & Mori folium \\
\hline 23 & Arachidonic acid & PPARG & Mori folium \\
\hline 24 & Tetramethoxyluteolin & ADORA2A & Mori folium \\
\hline 25 & Arteglasin A & $S L C 6 A 2, E S R 2$ & Flos Chrysanthemi Indici \\
\hline 26 & Daucosterol_qt & SLC6A2, ESR2 & Flos Chrysanthemi Indici \\
\hline 27 & Sitosterol & SLC6A2, ESR2 & Flos Chrysanthemi Indici \\
\hline 28 & Acacetin-7-O- $\beta$-D-galactopyranos & ADORA2A & Flos Chrysanthemi Indici \\
\hline
\end{tabular}

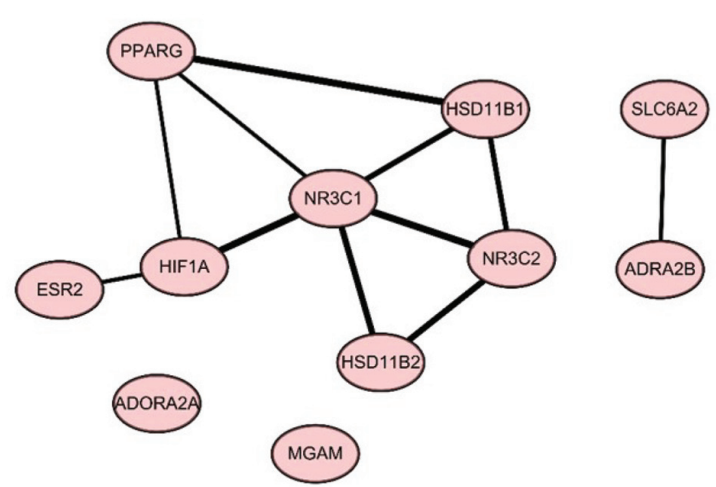

Fig. 4. XSJ and hypertension-related targets' protein-protein interaction network. components in TCM herbs have not yet been completely identified; thus, the databases of compounds are not complete, precluding their ability to represent the integral spectrum of compounds responsible for the anti-hypertension effect. Second, all of the data were based on silico analysis, and as such there may be many false positive and false negative interactions between the found compound-protein and protein-protein interactions. What's more, the relationship between XSJ and anti-hypertension activity was identified by enrichment analysis. Therefore, the associations presented herein should be further investigated for experimental verification to achieve more accurate and reliable inferences in the future.

Future directions

The associated biological processes and pathways need further 
Table 3. Hypertension-related targets of Xia Sang Ju

\begin{tabular}{|c|c|c|c|c|c|}
\hline No. & Target & Uniprot ID & Gene code & Protein class & Frequency \\
\hline 1 & Estrogen receptor-beta & Q92731 & ESR2 & $\begin{array}{l}\text { Nucleic acid binding; receptor; } \\
\text { transcription factor }\end{array}$ & 17 \\
\hline 2 & Solute carrier family 6 member 2 & P23975 & SLC6A2 & Transporter & 8 \\
\hline 3 & $\begin{array}{l}\text { Nuclear receptor subfamily } \\
3 \text {, group C, member } 1\end{array}$ & P04150 & $N R 3 C 1$ & $\begin{array}{l}\text { Nucleic acid binding; receptor; } \\
\text { transcription factor }\end{array}$ & 1 \\
\hline 4 & $\begin{array}{l}\text { Nuclear receptor subfamily } \\
3 \text {, group C, member } 2\end{array}$ & P08235 & NR3C2 & $\begin{array}{l}\text { Nucleic acid binding; receptor; } \\
\text { transcription factor }\end{array}$ & 1 \\
\hline 5 & Adenosine receptor $\mathrm{A} 2 \mathrm{a}$ & P29274 & $A D O R A 2 A$ & Receptor & 3 \\
\hline 6 & Adrenoceptor alpha 2B & P18089 & $A D R A 2 B$ & Receptor & 1 \\
\hline 7 & Maltase-glucoamylase & 043451 & MGAM & Hydrolase & 1 \\
\hline 8 & $\begin{array}{l}\text { Hypoxia-inducible factor } \\
1 \text {, alpha subunit }\end{array}$ & Q16665 & HIF1A & $\begin{array}{l}\text { Nucleic acid binding; } \\
\text { transcription factor }\end{array}$ & 1 \\
\hline 9 & $\begin{array}{l}\text { 11-Beta-hydroxysteroid } \\
\text { dehydrogenase, type I }\end{array}$ & P28845 & $H S D 11 B 1$ & None & 1 \\
\hline 10 & $\begin{array}{l}\text { Peroxisome proliferator- } \\
\text { activated receptor-gamma }\end{array}$ & P37231 & PPARG & $\begin{array}{l}\text { Nucleic acid binding; receptor; } \\
\text { transcription factor }\end{array}$ & 3 \\
\hline 11 & $\begin{array}{l}\text { Corticosteroid 11-beta- } \\
\text { dehydrogenase isozyme } 2\end{array}$ & P80365 & HSD11B2 & Oxidoreductase & 1 \\
\hline
\end{tabular}

investigation for confirmation of the exact mechanism of XSJ in hypertension treatment.

\section{Conclusions}

a

cellular nitrogen compound biosynthetic process transeription, DNA-templated positive regulation of nitrogen compound me tabolic process positive regulation of biosynthetic process positive regulation of cellular biosynthetic process nucleic acid-templated transcription positive regulation of nucleobase-containing compound metabolic process organic cyclic compound biosynthetic process aromatic compound biosynthetic process heterocycle biosynthetic process nucleobase-containing compound biosynthetic process
Biological process

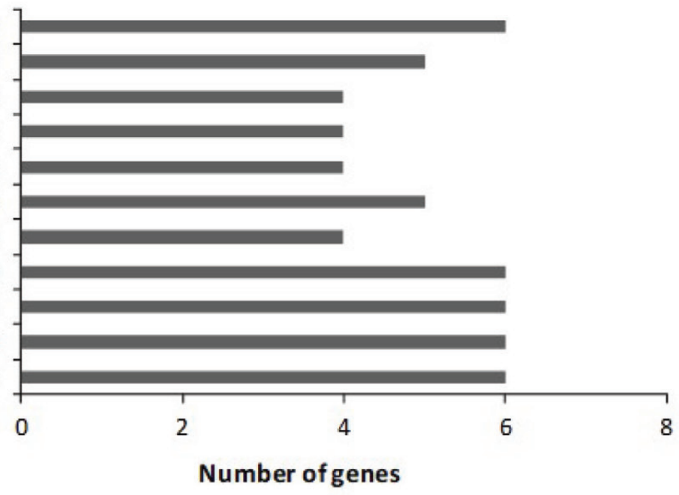

b KEGG pathway

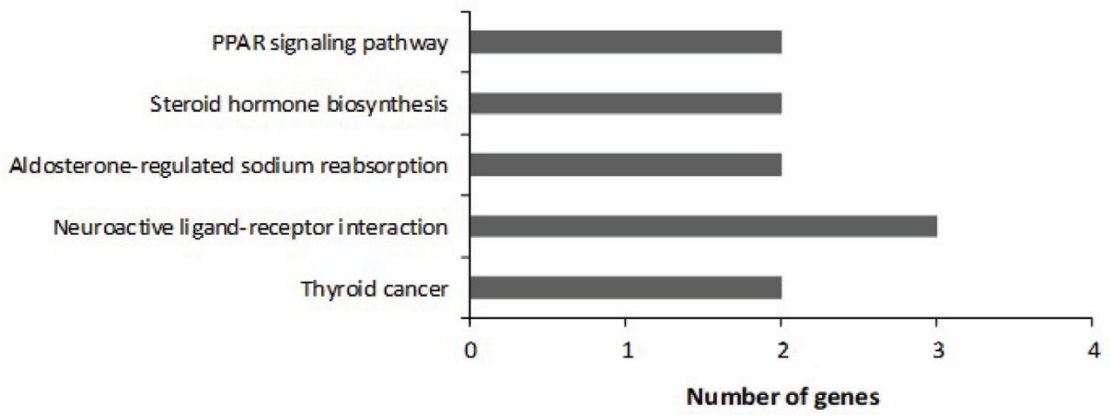

Fig. 5. Gene ontology functional analysis. (a) Biological processes terms. (b) Significant KEGG pathways. 
Collectively, the findings presented herein suggest that the compounds in XSJ exert their anti-hypertensive effect via multiple biological processes, such as regulation of blood pressure, blood vessel remodeling, regulation of the nitric oxide biosynthetic process and so on, which is in accord with the TCM therapy concept of "multiple compounds-multiple targets-multiple effects". Though further experiments are needed to verify this finding, this study revealed the potential anti-hypertensive mechanism of XSJ from holistic and systematic perspectives by using network pharmacology.

\section{Supporting information}

Supplementary material for this article is available at https://doi. org/10.14218/JERP.2020.00008.

Table s1. Hypertension targets.

Table s2. Interaction of genes related to hypertension.

Table s3. Relationships between compounds and targets.

\section{Acknowledgments}

The author wishes to thank Zhong Xiaotian and Gao Jiansheng for valuable comments.

\section{Funding}

This study was supported by Grants from Shenzhen Overseas High-Caliber Peacock Foundation (KQTD2015071414385495).

\section{Conflict of interest}

None.

\section{References}

[1] Lin L, Xu ZD, Yao JX, Liu JY, Li C, Wang ZM. Quality standards for Xiasangju Granules (in Chinese). Chin Tradit Pat Med 2012;34(08):15001505.

[2] Lackland DT, Weber M A. Global burden of cardiovascular disease and stroke: hypertension at the core. Can J Cardiol 2015;31(5):569571. doi:10.1016/j.cjca.2015.01.009.

[3] Dustan HP, Roccella EJ, Garrison HH. Controlling hypertension. A research success story. Arch Intern Med 1996;156(17):1926-1935. doi:10.1001/archinte.1996.00440160034007.

[4] Peng M, Watanabe S, Chan K, He Q, Zhao Y, Zhang Z, et al. Luteolin restricts dengue virus replication through inhibition of the proprotein convertase furin. Antiviral Research 2017;143:176-185. doi:10.1016/j.antiviral.2017.03.026.

[5] Hopkins AL. Network pharmacology. Nat Biotechnol 2007;25(10): 1110-1111. doi:10.1038/nbt1007-1110.

[6] Hopkins AL. Network pharmacology: the next paradigm in drug discovery. Nat Chem Biol 2008;4(11):682-690. doi:10.1038/nchembio.118.

[7] Zhang R, Zhu X, Bai H, Ning K. Network Pharmacology Databases for Traditional Chinese Medicine: Review and Assessment. Front Pharmacol 2019;10:123. doi:10.3389/fphar.2019.00123.

[8] Casas Al, Hassan AA, Larsen SJ, Gomez-Rangel V, Elbatreek M, Kleikers PWM, et al. From single drug targets to synergistic network pharmacology in ischemic stroke. PNAS 2019;116(14):7129-7136. doi:10.1073/pnas.1820799116.

[9] Ma C, Xu T, Sun X, Zhang S, Liu S, Fan S, et al. Network Pharmacology and Bioinformatics Approach Reveals the Therapeutic Mechanism of Action of Baicalein in Hepatocellular Carcinoma. Evid Based Complement Alternat Med 2019;2019:7518374. doi:10.1155/2019/7518374.

[10] Doak BC, Over B, Giordanetto F, Kihlberg J. Oral Druggable Space beyond the Rule of 5: Insights from Drugs and Clinical Candidates. Chem Biol 2014;21(9):1115-1142. doi:10.1016/j.chembiol.2014.08.013.

[11] Ru J, Li P, Wang J, Zhou W, Li B, Huang C, et al. TCMSP: a database of systems pharmacology for drug discovery from herbal medicines. $J$ Cheminform 2014;6:13. doi:10.1186/1758-2946-6-13.

[12] Walters WP, Murcko MA. Prediction of 'drug-likeness'. Adv Drug Deliv Rev 2002;54(3):255-271. doi:10.1016/s0169-409x(02)00003-0.

[13] Gfeller D, Grosdidier A, Wirth M, Daina A, Michielin O, Zoete V. SwissTargetPrediction: a web server for target prediction of bioactive small molecules. Nucleic Acids Res 2014;42(Web Server issue):W32W38. doi:10.1093/nar/gku293.

[14] Gfeller D, Michielin O, Zoete V. Shaping the interaction landscape of bioactive molecules. Bioinformatics 2013;29(23):3073-3079. doi:10.1093/bioinformatics/btt540.

[15] Amberger J, Bocchini C, Hamosh A. A new face and new challenges for Online Mendelian Inheritance in Man (OMIM $\left.{ }^{\circledR}\right)$. Hum Mutat 2011;32(5):564-567. doi:10.1002/humu.21466.

[16] Hu YA, Cui YX. Application of Online Mendelian Inheritance in Man to medical genetics (in Chinese). Zhonghua Nan Ke Xue 2011;17(7):639643.

[17] Stelzer G, Rosen N, Plaschkes I, Zimmerman S, Twik M, Fishilevich S, et al. The GeneCards Suite: From Gene Data Mining to Disease Genome Sequence Analyses. Curr Protoc Bioinformatics 2016;54:1.30.11.30.33. doi:10.1002/cpbi.5.

[18] von Mering C, Jensen LJ, Snel B, Hooper SD, Krupp M, Foglierini M, et al. STRING: known and predicted protein-protein associations, integrated and transferred across organisms. Nucleic Acids Res 2005;33(Database issue):D433-D437. doi:10.1093/nar/gki005.

[19] Warde-Farley D, Donaldson SL, Comes O, Zuberi K, Badrawi R, Chao P, et al. The GeneMANIA prediction server: biological network integration for gene prioritization and predicting gene function. Nucleic Acids Res 2010;38(Web Server issue):W214-W220. doi:10.1093/nar/ gkq537.

[20] Shannon P, Markiel A, Ozier O, Baliga NS, Wang JT, Ramage D, et al. Cytoscape: a software environment for integrated models of biomolecular interaction networks. Genome Res 2003;13(11):2498-2504. doi:10.1101/gr.1239303.

[21] Huang DW, Sherman BT, Lempicki RA. Systematic and integrative analysis of large gene lists using DAVID bioinformatics resources. Nat Protoc 2009;4(1):44-57. doi:10.1038/nprot.2008.211.

[22] Huang DW, Sherman BT, Lempicki RA. Bioinformatics enrichment tools: paths toward the comprehensive functional analysis of large gene lists. Nucleic Acids Res 2009;37(1):1-13. doi:10.1093/nar/gkn923.

[23] Hutson DD, Gurrala R, Ogola BO, Zimmerman MA, Mostany R, Satou $R$, et al. Estrogen receptor profiles across tissues from male and female Rattus norvegicus. Biol Sex Differ 2019;10(1):4. doi:10.1186/ s13293-019-0219-9.

[24] Zhao L, Fan X, Zuo L, Guo Q, Su X, Xi G, et al. Estrogen receptor 1 gene polymorphisms are associated with metabolic syndrome in postmenopausal women in China. BMC Endocr Disord 2018;18(1):65. doi:10.1186/s12902-018-0289-4

[25] Ogawa S, Emi M, Shiraki M, Hosoi T, Ouchi Y, Inoue S. Association of estrogen receptor beta (ESR2) gene polymorphism with blood pressure. J Hum Genet 2000;45(6):327-330. doi:10.1007/ s100380070002.

[26] Eikelis N, Marques FZ, Hering D, Marusic P, Head GA, Walton AS, et al. A polymorphism in the noradrenaline transporter gene is associated with increased blood pressure in patients with resistant hypertension. J Hypertens 2018;36(7):1571-1577. doi:10.1097/ HJH.0000000000001736.

[27] Zolk O, Ott C, Fromm MF, Schmieder RE. Effect of the rs168924 singlenucleotide polymorphism in the SLC6A2 catecholamine transporter gene on blood pressure in Caucasians. J Clin Hypertens (Greenwich) 2012;14(5):293-298. doi:10.1111/j.1751-7176.2012.00618.x. 
J Explor Res Pharmacol

[28] Yanagisawa $M$, Kurihara $H$, Kimura $S$, Tomobe $Y$, Kobayashi $M$, Mitsui $\mathrm{Y}$, et al. A novel potent vasoconstrictor peptide produced by vascular endothelial cells. Nature 1988;332(6163):411-415. doi:10.1038/332411a0.

[29] Halushka MK, Fan JB, Bentley K, Hsie L, Shen N, Weder A, et al. Patterns of single-nucleotide polymorphisms in candidate genes for blood-pressure homeostasis. Nat Genet 1999;22(3):239-247. doi:10.1038/10297.

[30] Gohlke P, Bünning P, Unger T. Distribution and metabolism of angiotensin I and II in the blood vessel wall. Hypertension 1992;20(2):151157. doi:10.1161/01.hyp.20.2.151.

[31] Joannides R, Haefeli WE, Linder L, Richard V, Bakkali EH, Thuillez C, et al. Nitric oxide is responsible for flow-dependent dilatation of human peripheral conduit arteries in vivo. Circulation 1995;91(5):13141319. doi:10.1161/01.cir.91.5.1314.

[32] Liang JQ, Xiong WN, Luo Y, Wang SS. Extract of Prunella vulgaris L. reduces blood pressure in essential hypertension rats (in Chinese). Journal of Chinese Medicine Materials 2011;34(1):99-100.

[33] Lu JP. An overview of the pharmacological effects of Mori Folium (in Chinese). Chinese Journal of Rural Medicine and Pharmacy 2013;20(12):83-84.

[34] Liu JF, Chu CC, Chien MK, Ting KS. Studies on antihypertensive drugs. XIII. Experimental therapy and toxicity of $\mathrm{HC}-1$, an extract from Chrysanthemum Indicum (in Chinese). Acta Pharmaceutica Sinica 1962;9(3):151-154.

[35] Wu YT, Chen L, Tan ZB, Fan HJ, Xie LP, Zhang WT, et al. Luteolin Inhibits Vascular Smooth Muscle Cell Proliferation and Migration by Inhib-
Peng M. Potential antihypertensive mechanism of XSJ

iting TGFBR1 Signaling. Front Pharmacol 2018;9:1059. doi:10.3389/ fphar.2018.01059.

[36] Su J, Xu HT, Yu JJ, Gao JL, Lei J, Yin QS, et al. Luteolin Ameliorates Hypertensive Vascular Remodeling through Inhibiting the Proliferation and Migration of Vascular Smooth Muscle Cells. Evid Based Complement Alternat Med 2015;2015:364876. doi:10.1155/2015/364876.

[37] Oyagbemi AA, Omobowale TO, Ola-Davies OE, Asenuga ER, Ajibade TO, Adejumobi OA, et al. Quercetin attenuates hypertension induced by sodium fluoride via reduction in oxidative stress and modulation of HSP 70/ERK/PPARY signaling pathways. Biofactors 2018;44(5):465-479. doi:10.1002/biof.1445.

[38] Pereira SC, Parente JM, Belo VA, Mendes AS, Gonzaga NA, do Vale GT, et al. Quercetin decreases the activity of matrix metalloproteinase-2 and ameliorates vascular remodeling in renovascular hypertension. Atherosclerosis 2018;270:146-153. doi:10.1016/j.atherosclerosis.20 18.01.031.

[39] Kim SG, Kim JR, Choi HC. Quercetin-Induced AMP-Activated Protein Kinase Activation Attenuates Vasoconstriction Through LKB1-AMPK Signaling Pathway. J Med Food 2018;21(2):146-153. doi:10.1089/ jmf.2017.4052.

[40] Parichatikanond W, Pinthong D, Mangmool S. Blockade of the reninangiotensin system with delphinidin, cyanin, and quercetin. Planta Med 2012;78(15):1626-1632. doi:10.1055/s-0032-1315198.

[41] Han S, Sung KH, Yim D, Lee S, Lee CK, Ha NJ, et al. The effect of linarin on LPS-induced cytokine production and nitric oxide inhibition in murine macrophages cell line RAW264.7. Arch Pharm Res 2002;25(2):170-177. doi:10.1007/BF02976559. 